\title{
EIN SPÄTLATÈNEZEITLICHER BEFUND IM PALAIS RASUMOFSKY, WIEN III, LANDSTRASSE (GRABUNG 2011)
}

\author{
PETER C. RAMSL ${ }^{10}-$ RA D OSLAV ČA M B A L
}

\begin{abstract}
A Late La Tène Period Object in Palais Rasumofsky, Vienna III, Landstraße (Excavation 2011). The present article is a processing of the finds of a La Tène period feature from Vienna III-Landstraße (Palais Rasumofsky). On 8 August 2011, excavation work began in the inner courtyard of the Palais Rasumofsky (Vienna III, Landstraße) due to the planned construction of an underground car park. During the work on the following days, extensive remains of a cellar in the north-eastern part of the area came to light. In addition, a fragment of pottery from the La Tène period was recovered from the excavated material. The subsequently excavated object was a so-called 'sunken hut' disturbed by walls on the northwest and southwest sides. It was preserved in a dimension of 2.5 by $2.3 \mathrm{~m}$ and contained six post pits. The maximum depth of the pit was $0.8 \mathrm{~m}$. The finds contain mainly of pottery, composed of broken pieces of vessels decorated with comb lines, undecorated pieces and a few painted pieces, mainly in the form of pots, bowls and individual pieces such as a lid and a tripod vessel. The find of two spindle whorls made of pottery is worth mentioning. In addition to pottery, the find material from the pit house included animal bones and a small number of iron and non-ferrous metal objects. Among the pottery, pots with thickened club rims and those with bottom marks stand out. The material dates to the Late La Tène period (LTD2) and can be compared well with that from Bratislava-Castle.
\end{abstract}

Keywords: Austria, Late La Tène Period, pit house, pottery.

Am 8. August 2011 wurde aufgrund des geplanten Baus einer Tiefgarage mit Baggerarbeiten im Innenhof des Palais Rasumofsky (Wien III, Landstraße) begonnen. Bei den Arbeiten an den folgenden Tagen traten umfangreiche Baureste eines Kellers im Nordostteil der Fläche zu Tage, wie auch ein Maueransatz an der südwestlichen Profilkante. Darüber hinaus konnte aus dem Aushubmaterial ein latènezeitliches Keramikbruchstück geborgen werden (Käferle/Schön 2011a).

Das latènezeitliche Material der Grube wurde vom Erstautor zur Bearbeitung übernommen, die Tierknochen kamen ins Naturhistorische Museum Wien, wo sie in der ersten Zoologischen Abteilung (Archäologisch-Zoologische Sammlung) von Dr. Konstantina Saliari und Dr. Erich Pucher bearbeitet wurden (Saliari/Pucher/Ramsl 2015). ${ }^{2}$
Schließlich wurden der Befund und die Funde in diesem Rahmen von Radoslav Čambal und Peter C. Ramsl bearbeitet.

\section{HISTORIE}

Fürst Andreas Kirillowitsch Rasumofsky, russischer Gesandter am Wiener Hof, erwarb nach seiner Ankunft in Wien im Jahr 1791 im Laufe mehrerer Jahre einige Grundstücke in der damaligen Rauchfangkehrer Gasse (Abb. 1). Er erwarb neben dem Gartenhaus und einem Teil des Gartens des berühmten Dr. Franz Anton Mesmer auch das anschließende Kottel'sche Haus sowie sechs kleinere bebaute Gründe mit den ehemaligen Konskriptionsnummern 254-260. 1803 reichte Fürst Rasumofsky

\footnotetext{
1 An dieser Stelle möchte ich dem Jubilar alles Gute, Gesundheit und vor allem Zufriedenheit wünschen. Unvergessen ist unsere langjährige Freundschaft, die vor unzähligen Jahren (es muss in den 1990ern gewesen sein) begann, als ich als junger Student das erste Mal in das Archäologische Institut in Nitra kam und von allen Kolleginnen und Kollegen herzlich aufgenommen wurde. Es folgten zahlreiche Begegnungen (mit intensivem wissenschaftlichen Austausch und auch gemütlichen Abendveranstaltungen) auf den jährlich veranstalteten Kelti/Keltové Tagungen der tschechisch-slowakischen Eisenzeitgruppe und schließlich mein dreijähriger Aufenthalt in Nitra (2016-2018) im Rahmen des SASPRO-Projektes 1340/03/03 „Male Identities in La Tène Cemeteries (MILT) in the Middle Danube Region”, wo Karol als mein "Scientist in Charge“ fungierte und sich auch sehr einsetzte, dass ich das Projekt schließlich bekommen konnte.

2 Für die Zurverfügungstellung des Materials sei dem Besitzer des Grundstückes, Mag. Adrian Riklin sowie Mag. Christoph Blesl (Bundesdenkmalamt, Abt. Archäologie) herzlich gedankt. Ebenso möchten wir den Ausgräberinnen, Mag. Doris Käferle und Mag. Doris Schön (AS-Archäologieservice) und dem Grabungsteam für die Grabungsdokumentation und zusätzliche mündliche Informationen danken. Dr. Konstantina Saliari und Dr. Erich Pucher sei für die Auswertung, Interpretation und Publikation der gefundenen Tierknochen gedankt.
} 


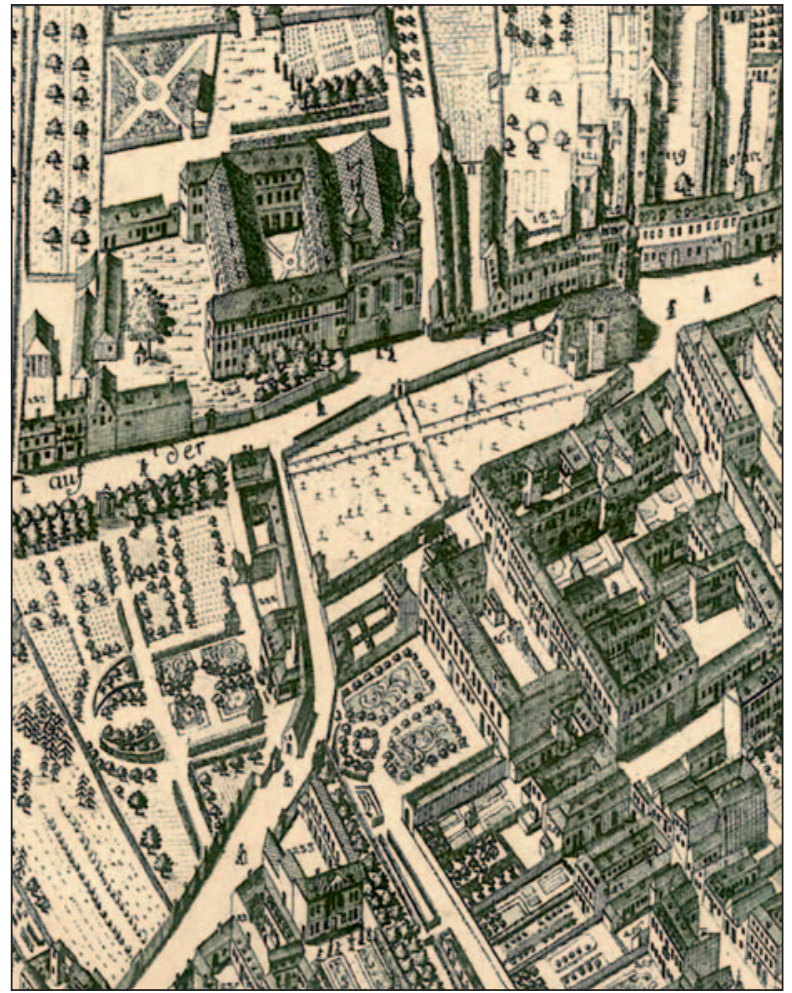

Abb. 1. Historische Darstellung des Palais Rasumofsky (Käferle/Schön 2011a, Abb. 2).

um Erteilung des Baukonsenses beim Wiener Magistrat ein, wobei mehrere Pläne von Joseph Meissl, der als Baumeister für die Bauarbeiten verantwortlich sein sollte, beigelegt waren. Die Pläne zeigen teilweise unterschiedliche Grundrissentwürfe und spiegeln den komplizierten Entstehungsprozess des Palais mit seinem angrenzenden Gartenhaus wider. Die Annahme Margarete Girardis, dass die Errichtung des Gebäudes zügig vorangeschritten sei und dass 1807 bereits große Teile standen ist demnach vehement anzuzweifeln (Girardis 1937). Am 31. Dezember 1814 brannte der Gartentrakt ab, wobei wertvolle Kunstschätze wie die Bibliothek verloren gingen (Käferle/Schön 2011b).

\section{GRABUNG}

Im Westbereich des Baggerschnittes kam der Befund eines latènezeitlichen Objekts zutage, der jedoch im Westen sowie im Süden von den Ziegelmauern gestört wurde. Dieser Befund hatte eine noch erhaltene Grundfläche von etwa 2,5×2,3 m und wies annähernd die Form eines Viertelkreises auf, wobei im Osten eine leicht abgerundete Erweiterung festzustellen war. Die Wände waren nach innen gekippt und der Übergang zum fast flachen Boden abgerundet. Das Objekt wies eine maximale Tiefe von ca. 0,80 bis $0,85 \mathrm{~m}$ auf, wobei aufgrund der Störung durch Ziegelmauern und der Baggerarbeiten das Interface nur eine Tiefe von $0,5 \mathrm{~m}$ zeigte. Die obersten Verfüllungen waren mittelgraubraun bis dunkelgraubraun und enthielten neben Kieseln etwas Holzkohle und vor allem Hüttenlehm. Die Schicht SE 90 bedeckte eine "Scherbenlage“ mit den Resten eines größeren Gefäßes (Abb. 2). Die folgende Schicht (SE 96) ist gekennzeichnet durch eine hell- bis mittelbraune Farbgebung und wenigen gelben Lehmflecken sowie etwas gebrannten Lehm und Holzkohle. Nach Abbau dieser Schicht war im Osten bereits der gewachsene Boden erreicht. In der restlichen Fläche überlagerten sich die Befunde zweier sehr dünner hell- bis mittelgraubrauner Schichten mit Holzkohle und wiederum etwas gebranntem Lehm (SE 98, 104), wobei die letzte Schicht (SE 104) auch einen hohen Anteil an Asche aufwies und insgesamt sechs Pfostenlöcher überdeckte (IF 113-118), die sehr unterschiedliche Formen und Tiefen aufwiesen. Die Pfostenlöcher 113-116 lagen in einer zirka NordostSüdwest verlaufenden Reihe in der Westhälfte des erhaltenen Objektes. Die Pfostenlöcher 117 und 118 befanden sich östlich annähernd parallel dazu jedoch leicht nach Nordosten versetzt. Zur etwaigen Innenausstattung des Grubenhauses liegen keine weiteren Befunde vor. Bei dem vorliegenden Objekt handelt es sich nach erster Beurteilung um den Rest eines latènezeitlichen Grubenhauses.

Die Keramik setzt sich vor allem aus kammstrichverzierten Gefäßbruchstücken, unverzierten sowie wenigen bemalten Stücken zusammen, die hauptsächlich in Form von Töpfen, Schüsseln und in Einzelstücken wie einem Deckel und einem Dreifußgefäß vorliegen. Darüber hinaus ist der Fund zweier Spinnwirtel aus Keramik erwähnenswert. Neben Keramik umfasste das Fundmaterial des Grubenhauses Tierknochen, Hüttenlehm und in geringer Anzahl Eisen- sowie Buntmetallobjekte.

\section{Befund}

Bei dem ergrabenen Objekt handelt es sich um eine eingetiefte Hütte, die an der Nordwest- und Südwestseite von Mauern gestört war (Abb. 3). Sie war in einer Dimension von 2,5 $\times 2,3$ m erhalten und enthielt sechs Pfostengruben. Die maximale Tiefe der Grube betrug 0,8 $\mathrm{m}$.

Eintiefungen größerer Dimension in annähernd rechteckiger Form werden in der Eisenzeit meist als Eingetiefte Hütten (also Bauten, wo das Dach mehr oder weniger direkt an den Boden anschließt) oder auch als Grubenhäuser bezeichnet. Diese sogenannten Grubenhäuser kommen beispielsweise im Karpatenbecken nicht nur in der gesamten Eisenzeit, 


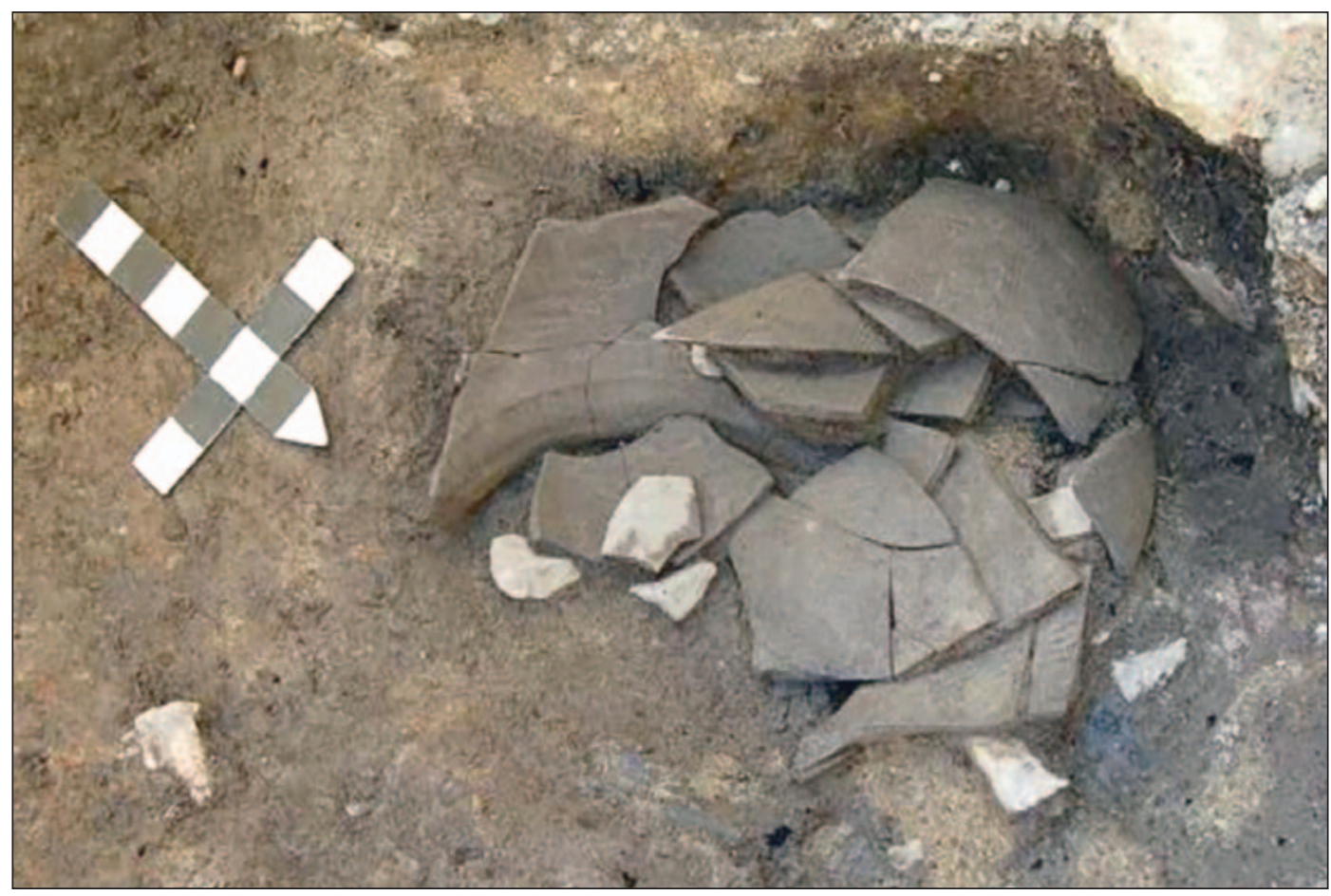

Abb. 2. Wien III, Palais Rasumofsky, latènezeitliches Grubenhaus. Scherbenlage in der obersten Verfüllung (Foto Käferle/Schön, AS, BDA).

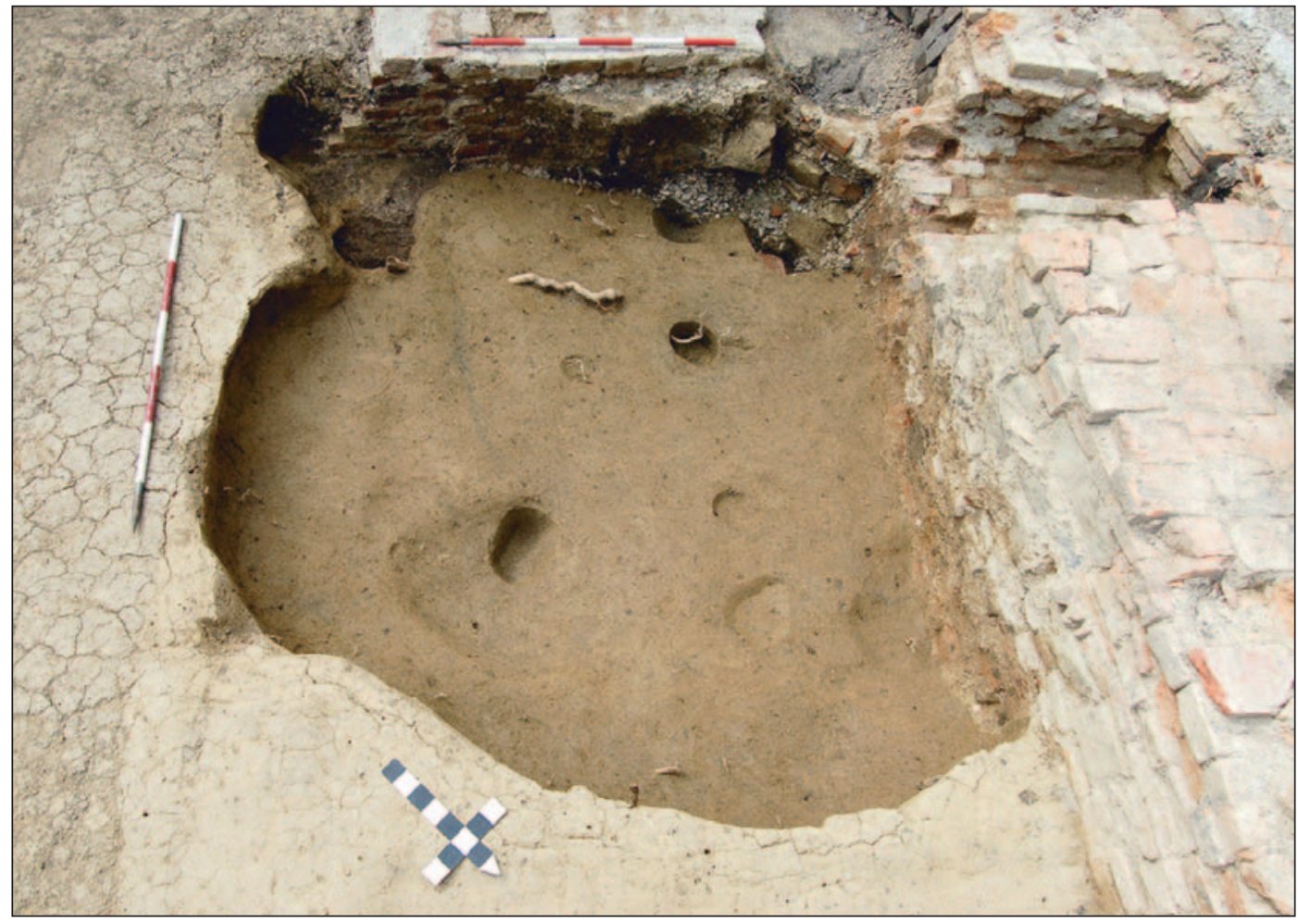

Abb. 3. Wien III, Palais Rasumofsky. Gesamtaufnahme des Grubenhauses (Foto Käferle/Schön, AS, BDA). 
sondern auch im Mittelalter und in der Neuzeit vor (Timár 2016, 191). Innerhalb der Grubenhäuser kann zwischen verschiedenen Konstruktionstypen unterschieden werden und es wurden eine Reihe von Typologien aufgestellt (z. B. Karl 1996, 56-72; Meduna 1980, 48; Waldhauser u. AG 1993, 348, Abb. 146).

\section{AUSWERTUNG DER KERAMIK}

Aus der Lokalität stammen zahlreiche Fundstücke in Form von Keramikfragmenten. Sie sind typische Formen für die Spätlatènezeit. Die zahlreichsten Analogien sind aus dem Gebiet des Oppidums von Bratislava bekannt.

\section{Situlentöpfe}

Auf der Fundstelle sind am häufigsten Situlentöpfe mit einem verstärkten Rand der Gruppe I vertreten (Taf. I: 1-3, 5; II: 9). Sie treten sowohl mit als auch ohne Graphit im Ton auf oder nur mit graphitierter Oberfläche. Zu dieser Gruppe von Gefäßen gehört auch eine Situla mit einer verstärkten Kante und einer Rille auf der Oberseite (Taf. I: 2). Dazu gehört auch ein großes graues Vorratsgefäß mit einem metopen-artigen Kammstrich auf der Oberfläche und mit einem Reparaturloch (Taf. III: 8). Ähnliche Töpfe kamen bei Ausgrabungen in Bratislava am Námestie Slobody Platz zutage, als ein Zweikammern - Töpferofen untersucht wurde. Leider sind sie bis jetzt nicht analysiert worden (Janšák 1955, 217, 218, Abb. 2; 3). Alle Typen haben zahlreiche Analogien in spätlatènezeitlichen Oppida und Siedlungen in Mitteleuropa. Diese Randformen gehören zeitlich in die Stufen LTC2 bis LTD1 und LTD2 (Bednár/Březinová/Ptáčková2005, 145, 146; Bónis 1969; Březinová 2000; Březinová/Chropovský 2020, 57-61; Čambal 2004, 27; Čambal/Kovár/Hanuš 2012, 120; Pieta/Zachar 1993, 157; Trebsche 2010; 2011).

\section{Töpfe mit kolbenförmig verdickten, gerade oder schräg abgestrichenen Rändern}

Zu einer Gruppe nächst den sogenannten Töpfen mit kolbenförmig verdickten, gerade oder schräg abgestrichenen Rändern gehört ein Exemnplar, dass ohne die Hilfe einer Töpferscheibe hergestellt wurde. Seine Oberfläche ist mit einem schrägen Kammstrich bedeckt, der typisch für die späten Formen der spätlatènezeitliche Keramik ist (Taf. I: 7). Der Typ liegt in der Nähe der Gruppe der Töpfe vom Typ I/2, die im Gebiet der südwestlichen Slowakei mit dem Zentrum in Bratislava und Umgebung vorkommen, wo diese spezifische Form während der Stufe LTD2 auftritt und zu den gängigen Keramiktypen im Bratislaver Oppidum sowie an mehreren Orten im mittleren Donauraum gehört (Čambal et al. 2016, 147-164, Abb. 1: 2-4). Es gibt ihn sowohl scheibengedreht als auch nur frei mit der Hand geformt. Die näheren Analogien stammen aus dem Grubenhaus 3/78 in Senec-St. Martin (Čambal/Mináč/Zachar 2010, 143, 145, Taf. I: 20, 21, 23). Sie treten auch in Österreich, in der Steiermark und auch selten in Slowenien auf. Es war ein Gebiet, das mit den Stämmen der Taurisci und Noriker in Verbindung gebracht werden kann. Hierher gehören die Funde von Södingberg bei Köflach, Lebing und Frauenberg bei Leibnitz (Čambal et al. 2016, 149, Abb. 5; 6). W. Artner erwähnt diese Art von Topf als eine der führenden Formen der Stufe LTD2 (Artner 1998-1999).

\section{Verzierungsmotive auf der Keramik}

Das charakteristischste dekorative Element der keltischen Keramik, insbesondere an der Situlatöpfen, ist der sogenannte „Kammstrich“. Es erscheint manchmal in Kombination mit schrägen Rillen (Taf. I: 1) oder mit metopenartigem Kammstrich in schräger Ausführung, typisch für mittelgroße graue Vorratsgefäße (Taf. I: 9). Die Kombination von vertikalem und schrägem Kammstrich wird als junges Element bei der Dekoration von Keramik angesehen, die hauptsächlich in der späten Latènezeit auf oppidaler Keramik auftritt (Čambal 2004, 22; Hlava/Vích 2007, 37). In ähnlicher Weise sind die jungen Elemente bei der Dekoration von Gefäßen durch vertikalem und metopenartigem Kammstrich (Taf. I: 4). Zum vorliegenden Inventar gehört auch eine Scherbe mit eingeglätteter Dekoration in Form eines schrägen Gitters (Taf. II: 12), die andererseits auch typisch für graue Keramik mit vasenartigen Formen ist. Diese Dekorationsarten und ihre gegenseitigen Kombinationen gehören zum Kanon der spätlatènezeitlichen Stufen LTD1a und LTD2 (Čambal/Kovár/Hanuš 2012, 121).

\section{Bodenzeichnen}

Aus der Fundstelle stammen zwei verschiedene Situlenböden mit Bodenzeichnen. Bodenzeichen treten einerseits auf Graphitkeramiken auf und grundsätzlich nur auf situlenförmigen Töpfen. Nach der Typologie von I. Kappel (1969, Abb. 52), resp. von M. Čižmár und J. Meduna (1985, Abb. 3: 1-5) gehört das erste Bodenzeichen (Taf. I: 1) zur Gruppe 7, das zweite Exemplar (Taf. II: 9) ist eine Kombination der Gruppen 7 und 11. Beide sind auch aus dem Oppidum Bratislava-Vydrica bekannt (Čambal/Kovár 2019, Taf. II: $4 a-c)$. Die Bodenzeichen auf den Situlentöpfen sind grundsätzlich nur mit Graphitmagerung verbunden, und ihre Herstellung ist eng mit dem Gebiet Böhmen, 
Mähren, Österreich und Bayern verbunden. In der Spätlatènezeit (LTD1 und LTD2) gibt es Bodenzeichen auch auf Keramik ohne Graphitmagerung im Oppidum von Bratislava und aus der Flachsiedlung in Kúty. Es wird davon ausgegangen, dass es sich um Marken einzelner Produktionszentren oder Werkstätten handelt, in denen diese Art von Töpfen hergestellt wurde (Hlava 2008, 216). Eines der Produktionszentren für Graphitonkeramik mit Bodenzeichen war Staré Hradisko in Mähren, wo Graphitsitulen mit verschiedenen Zeichen der Typen 6a, 6b, 9 und 11 hergestellt wurden (Čižmář/Meduna 1985, Abb. 4; 6; Hlava 2008, 216), ebenso in Manching (Kappel 1967). Bei den Funden aus dem Gebiet der Stadt Bratislava kennen wir derzeit 14 Arten von Bodenzeichen (Čambal/Kovár 2019, 180-182; Vrtel 2012, 170). Es ist wahrscheinlich, dass solche Bodenzeichen am Ende von LTC2 auftreten, ihr größtes Auftreten ist jedoch im LTD1 (Čižmářl Meduna 1985, 93, 94; Kappel 1969, 117). Ist es jedoch wahrscheinlich, dass die Bodenzeichen noch in der Stufe LTD2 auftreten, was aber mit der Frage verbunden ist, ob sie zu diesem Zeitpunkt noch produziert wurden oder nicht (Čambal/Kovár 2019, 182).

\section{Dreifußgefäße}

Ein rekonstruierbares Exemplar dieser Keramik stammt ebenfalls aus dieser Fundstelle. Topfartige Dreifüße der Gruppe VI (Taf. III: 7) werden auf einer Töpferscheibe aus Ton mit oder ohne Beimischung von Graphit hergestellt. Sie haben eine typische abgerundete Kante und einen relativ niedrigen Körper. Dreifüße werden auch innerhalb der Funde von Bratislava eindeutig auf den Überganghorizont LTD/LTD2, besonders nach LTD2 datiert (Čambal 2004, 29, Taf. XIV: 7; LXI: 9, 10; LXII: 2; Čambal et al. 2006, 141, Taf. III: 5a, b; V: 7; Musilová/Lesák 1996, Taf. III: 5, 6; Pieta 1996, 185, Abb. 1: 10, 11, 13; Pietal Zachar 1993, 161, Abb. 91: 1-5; Urban 1996; Zachar 1982, Abb. 6; Zachar/Rexa 1988, Abb. 17: 8). Gleiches gilt für Nitriansky Hrádok (Pieta 1996, Abb. 1: 12). Sie wurden zuletzt auch im 3. Wiener Bezirk (Landstraße) gefunden (Adler-Wölfl 2012, 168, Abb. 4: 2).

\section{Schüsseln}

Die zahlreichste Gruppe neben den situativen Töpfen in den Funden der Mittel- und Spätzeit sind die konischen La Tène-Schalen Typ V/2 mit einer nach innen gezogenem Rand (Taf. II: 8). Sie gehören jedoch nicht zu chronologisch empfindlichen Formen und haben zahlreiche Analogien in allen La Tène-Siedlungen in ganz Mitteleuropa (Čambal 2004, 19, 27, 28; Cambal/Kovár/Hanuš 2012, 120).

\section{Kelchförmige Becher}

Ein fassförmiges Gefäß mit einem geraden, nicht verstärkten Rand vom Typ IV tritt im vorliegenden Material nur in einem Fall in Form eines Fußfragments auf (Taf. II: 7). Sie kommen im Oppidum von Bratislava häufig als Teil feintoniger Tischkeramik vor (Musilová/Lesák 1996, 87 ff.). Es ist eine Keramik, die zum Materialkanon der Stufe LTD2 gehört (Čambal 2004, 18, 19, Taf. XLII; Čambal/Kovár/Hanuš 2012, 121; Pieta/Zachar 1993, Abb. 108; Zachar/Rexa 1988, 60, Abb. 19). Direkte Analogien stammen aus Wien ebenfalls vom Rochusmarkt (Adler-Wölfll Mosser 2015, 21, 22, Abb. 9: rechts)

\section{Flaschen/Vasen}

Der Hals eines dünnwandigen Flaschen-/Vasengefäßes der Gruppe II/1 stammt ebenfalls aus der Lokalität (Taf. III: 4). In diesem Fall handelt sich um weiße Flächenbemalung, was typisch für diesen Gefäßtyp ist. Bei dieser Art von Keramik wurden häufig horizontale Streifen unterschiedlicher Breite auf den Körper gemalt. Vorwiegend wurden rote, orange und weiße Engoben verwendet. Es ist eine typisch spätlatènezeitliche Form, die zur feinen Tischkeramik gehört. Im Oppidum von Bratislava finden sich zahlreiche Analogien (Čambal 2004, 25, Taf. XXXIV; XXXV; Čambal et al. 2015, 228, 229, Abb. 3; Čambal/Kovár/Hanuš 2012, 121, 122; Pieta/Zachar 1993, 157). Zu einer spätlatènezeitlichen Flasche gehört auch der Unterteil mit Boden (Taf. II: 11).

\section{Bemalte Keramik}

Ein Teil des Keramikinventars sind bemalte Scherben. Es ist eine Kombination aus weißer, orangefarbener und roter Malerei in horizontalen Streifen (Taf. I: 8; III: 1). Sie gehören zu den Flaschen der Gruppe II. Schwarz gemalte Gittermotive (Taf. I: 6), weisen wiederum Analogien im Fundmaterial aus der Oppidum Bratislava auf, wo sie im Sepia Farbton aufgetragen wurden (Čambal 2004, 24; Čambal/Gregor 2008, 97-106, Taf. I; II) und selten mit schwarzer Farbe (Čambal 2004, Taf. LXIII: 1, 2; LXXIV). Es gab auch eine relativ dickwandige Scherbe mit zwei horizontalen Bändern, die in grau-schwarz gehalten waren (Taf. III: 2). Aus Wien vom Rochusmarkt (Wien III) stammt eine ähnliche Bemalung mit grauer Malerei (AdlerWölfl/Mosser 2015, 22, Abb. 9: links). Es gibt auch ein schwarzes Motiv in Form eines Schachbretts und vertikaler Bänder auf weißem Hintergrund (Taf. III: 3) ähnlich wie in Manching (Maier 1970, Taf. 108: 11). Auch ein weiteres Flaschen-/Vasenfragment 
mit Bemalung, wo ein mehrmaliges Girlandenmotiv bzw. ein Halbmondmuster in brauner Farbe auf weisen Hintergrund, mit roten und orangenen Streifen (Taf. III: 6) auftritt, hat Parallelen in Manching (Maier 1970, 89, 90, Taf. 89: 1234-1237). Möglicherweise handelt es sich in diesem Fällen um Manchinger Importe. Die Flaschen/Vasen vom Typ II/1 und II/3 sind ein typischer Bestandteil des spätlaténezeitlichen Materials der Oppidazeit. Ein ähnliches Halbmondmotiv ist auch aus Bratislava bekannt (Pietal Zachar 1993, Abb. 90: 6). Es gibt sowohl bemalte als auch unbemalte Exemplare davon. Während der Spätlatènezeit konzentrierte sich ihre Produktion auf Zentren wie Manching (Maier 1970), Stradonice, Třísov, Staré Hradisko (Cumberpatch 1993, 81 ff.) und Bratislava (Čambal 2004, 23-27, Taf. LXVIII-LXXVI; Čambal et al. 2015, 228-229; Abb. 3: 1, 3, 7, 8, 11; Cumberpatsch 1993, 60). Ein Fragment eines topfförmigen, dünnwandigen Gefäßes mit Malerei hat breite weiße horizontaler Streifen auf orangefarbenen Hintergrund (Taf. I: 10) und ist ebenfalls eine typische spätlaténezeitliche Form. Interessant ist, dass es ein sekundär gebohrtes Loch besitzt, welches mit Blei verstopft ist. Wahrscheinlich handelt es sich um ein Reparaturloch, welches üblicherweise mit eisernen Klammern repariert wurde. Solche gebohrten Reparaturlöcher treten häufig bei Siedlungskeramik auf. Mit Hilfe von Eisen- und selten Bronzeklammern wurden hauptsächlich Graphitton-Situlentöpfe, aber auch andere Gefäßformen repariert (Čižmár 2003, 57; Meduna 1980, 110, 111).

\section{Deckel}

Ein Stück ist auf dieser Fundstelle durch einen größeren konischen Deckel Gruppe VIII/1c vertreten (Čambal 2004, 26). Es handelt sich um ein Exemplar mit versetztem Innenrand größerer Form, auf der Töpferscheibe gedreht, aus fein geschlämmtem Ton in hellbrauner und dunkelgrauer Farbe (Taf. III: 5). Diese Art der Deckel wurde wahrscheinlich für einige der Varianten kleiner und mittlerer Töpfe verwendet. Die Deckeloberfläche wurde sorgfältig ohne Spuren von Malerei mit Engobe behandelt. Exemplare davon erscheinen auch in Wien am Leopoldsberg (Urban 1999, Abb. 63: 442). Sie kommen hauptsächlich im 1. Jahrhundert vor Christus vor, d. h. sie gehören zum Materialkanon der Stufe LTD1 und insbesondere LTD2. Sie werden häufig auch rot bemalt. Üblich sind z. B. im Oppidum von Bratislava und ihrem Hinterland (Čambal 2004, 26, 29, Taf. LVII; Čambal et al. 2015, 228, Abb. 3: 7, 8; Pietal Zachar 1993, Abb. 90: 4; Urban 1996, Abb. 1: 4; 1999, 221; Zachar 1981, Abb. 10: 13; 1982, 173: 4; Zachar/Rexa 1988, Abb. 17: 9).

\section{Spinnwirtel}

Weitere Keramikfunde aus der Fundstelle sind Spinnwirteln, die aus latènezeitlichen Situlentopfscherben mit Kammstrich auf der Oberfläche hergestellt wurden (Taf. II: 5, 6). Spinnwirteln dieser Art gehören zu den häufigsten Funden in Siedlungen aus dem Mittel- und Spätlatènezeit (LTC2 bis LTD2) in ganzes Mitteleuropa. Dies weist auf eine entwickelte eigene häusliche Webproduktion hin (Březinovál Chropozský 2020, 61). Für die Chronologie der mittleren- und späten Latènezeit haben diese Funde (durch ihre Einheitlichkeit) keine wichtige Bedeutung - außerdem wurden sie ja aus älteren latènezeitlichen Scherben gefertigt (Čambal 2004, 30, 31, Taf. LXXV).

Zusammenfassend kann gesagt werden, dass die Keramik aus der Rasumofskygasse in die spätlatènezeitlichen Stufen LTD1 und LTD2 datiert werden kann. Die wahrscheinlich ältesten Exemplare sind die Situlentöpfe mit Bodenzeichen, die nach LTD1 datierbar sind, mit einer Überlappung bis LTD2. Andere Formen von Tongefäßen gehören in die Stufe LTD2, einschließlich auch der Situlentöpfe, Flaschen, Deckel sowie bemalter Keramik. Eines der chronologisch jüngsten Stücke ist das handgemachte Gefäß aus der Gruppe der sogenannten Töpfe mit kolbenförmig verdickten, gerade oder schräg abgestrichenen Rändern, die nach LTD2 datieren.

\section{Andere Kleinfunde}

Neben den Keramikfunden konnten auch weitere Kleinfunde aus Buntmetall, Eisen und Knochen gemacht werden.

Aus Buntmetall sind ein Draht (Taf. II: 3), ein rinnenförmiges Objekt (Taf. II: 2) sowie ein halbkugelförmiges Artefakt (Taf. II: 1), die nicht einer bestimmten Funktion zugeordnet werden können. Eventuell kann das halbkugelförmige Artefakt als Nagelkopf bezeichnet werden. Daneben liegen noch einige undefinierte nicht abgebbildete Fragmente aus Eisen vor.

Aus Knochen ist ein bemerkenswertes Artefakt (Taf. II: 4). Es handelt sich dabei um ein geschnitztes, schmales, stiftförmiges Objekt mit rechteckigem Querschnitt und seitlich abstehenden Fortsätzen (im oberen Bereich zwei, im mittleren einer), die vermutlich abgerochen sind. Die Funktion ist unbekannt.

\section{ZUSAMMENFASSUNG}

Der Fundort im Palais Rasumofsky reiht sich gut in die bisher bekannten Auffindungen in Wien Landstraße ein (Abb. 4). Bei dem beschriebenen Befund handelt sich um eine eingetiefte Hütte 


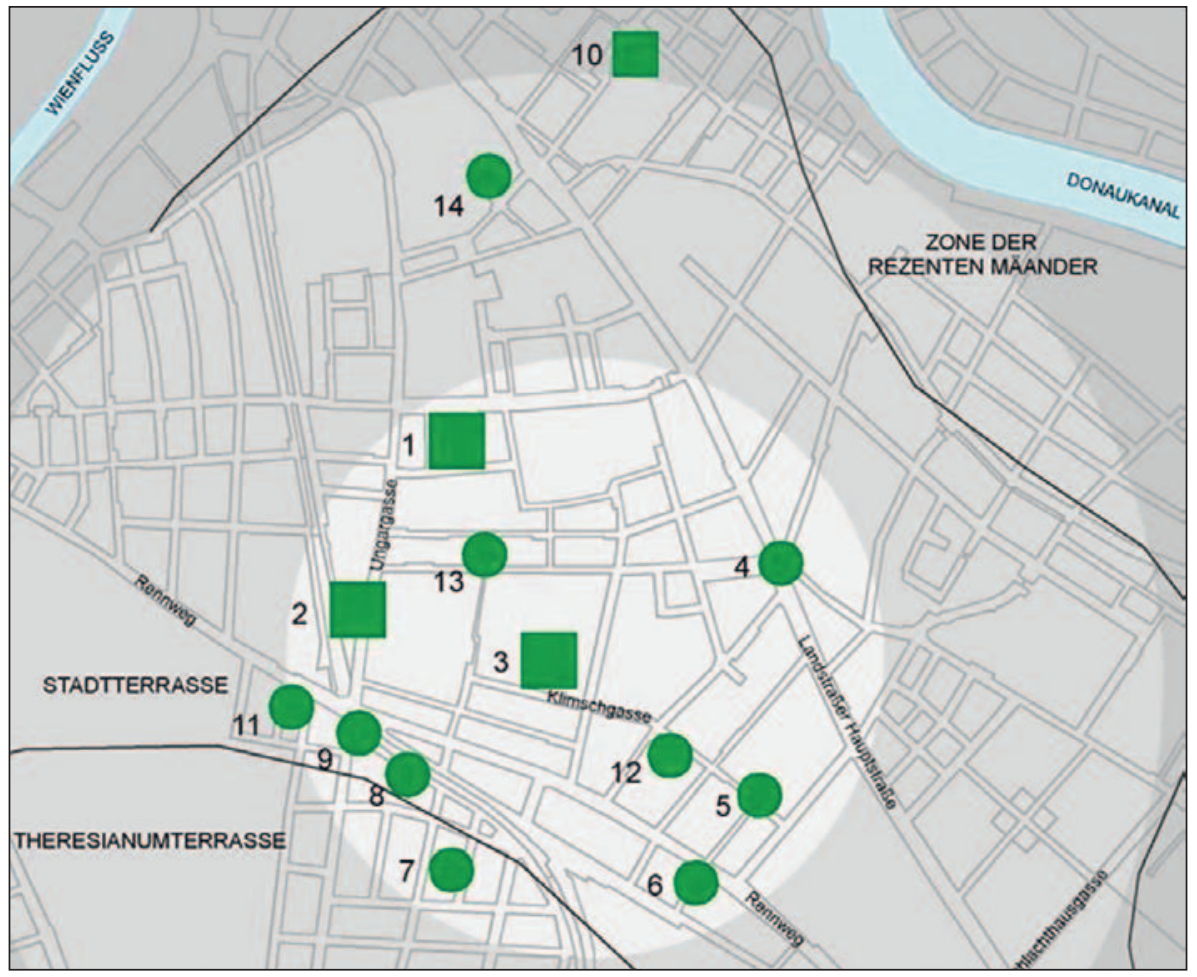

Abb. 4. Latènezeitliche Fundstellen in Wien III, Palais Rasumofsky als Nr. 10 (ergänzt nach Donat/Pichler/Sedlmayer 2002, Abb. 4).

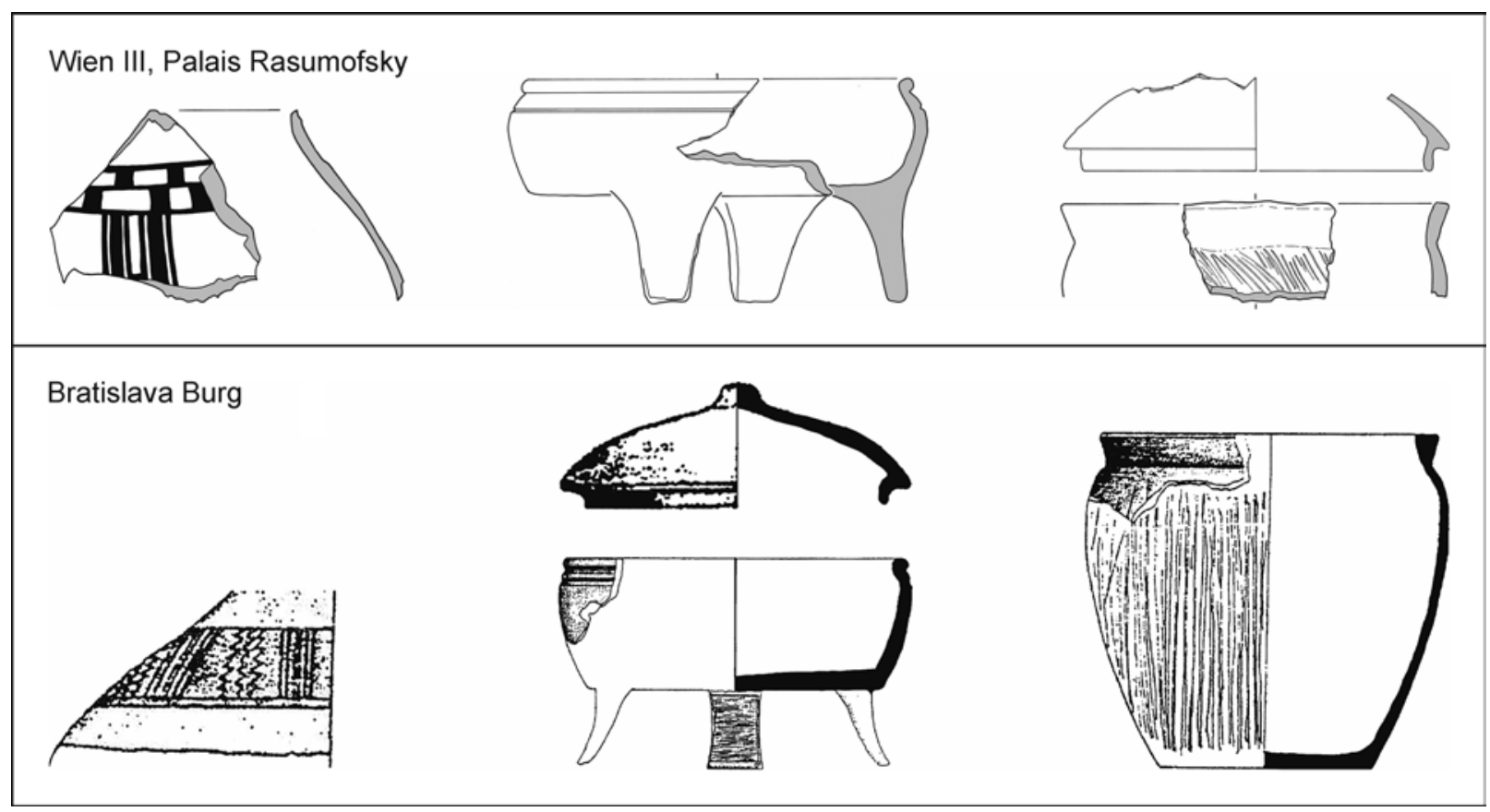

Abb. 5. Vergleich der Funde (P. C. Ramsl, ergänzt nach Čambal 2004, Taf. LXVIII).

mit Spuren von Pfostenlöchern. Die Funde darin (Keramik und einige wenige Kleinfunde aus Buntmetall, Knochen und Eisen) datieren in die
Spätlatènezeit. Das vorgestellte Material kann gut mit dem von Bratislava Burg verglichen werden, wie auf Abb. 5 zu sehen ist. 


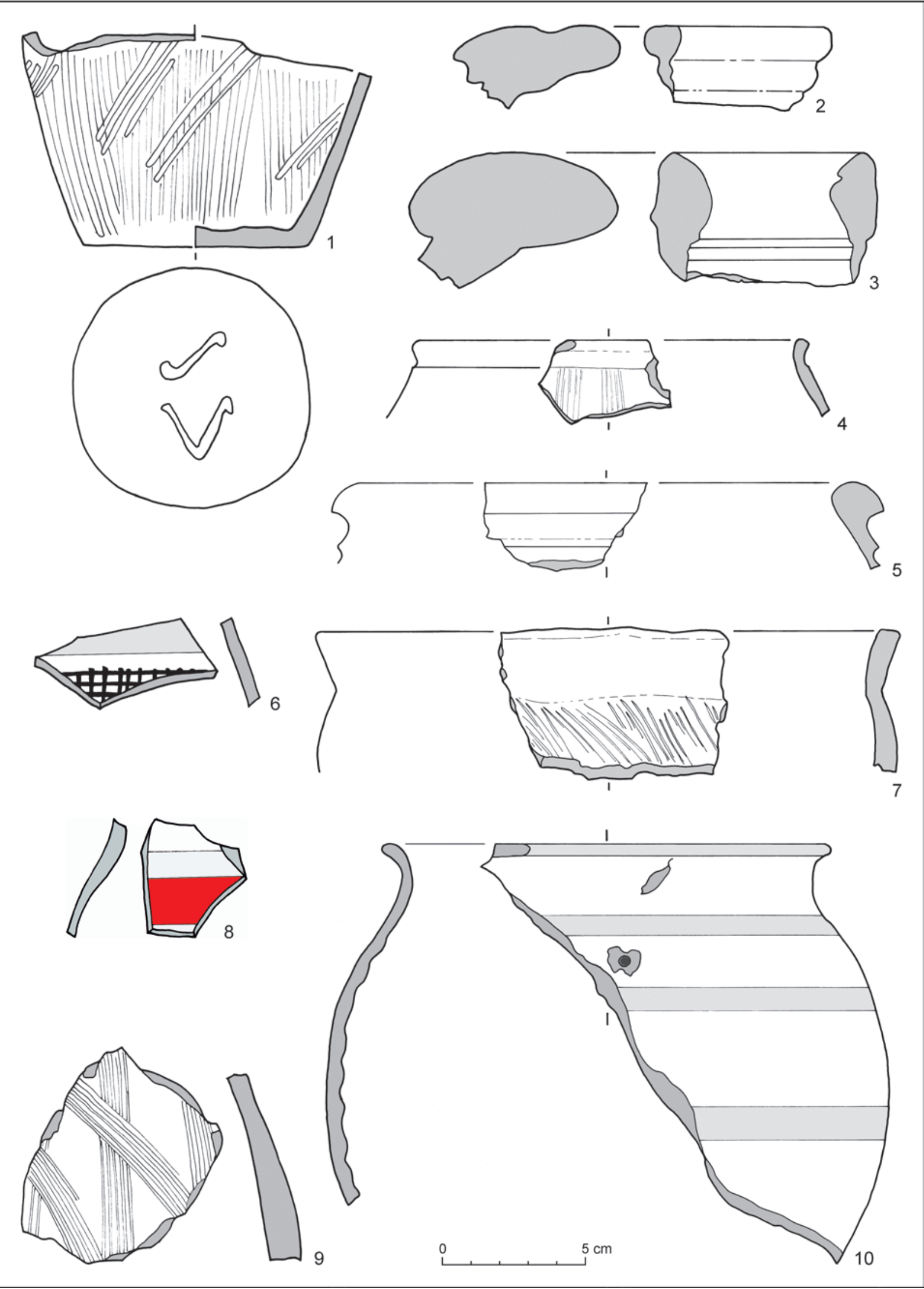

Taf. I. Wien III, Palais Rasumofsky, latènezeitliches Grubenhaus. Keramikfunde. 


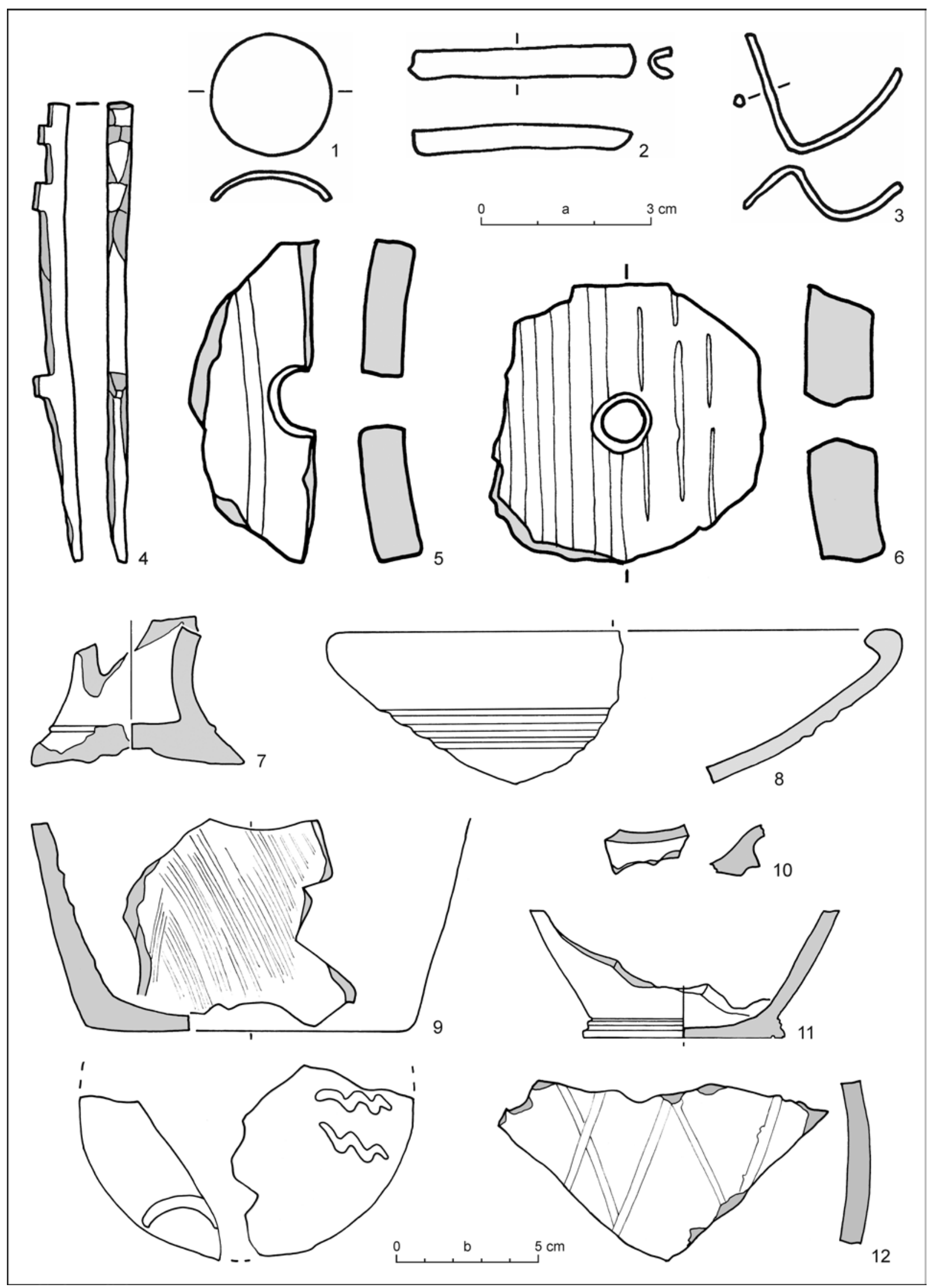

Taf. II. Wien III, Palais Rasumofsky, latènezeitliches Grubenhaus. 1-6 - Kleinfunde; 7-12 - Keramikfunde. Maßstab: $\mathrm{a}-1-6 ; \mathrm{b}-7-12$. 

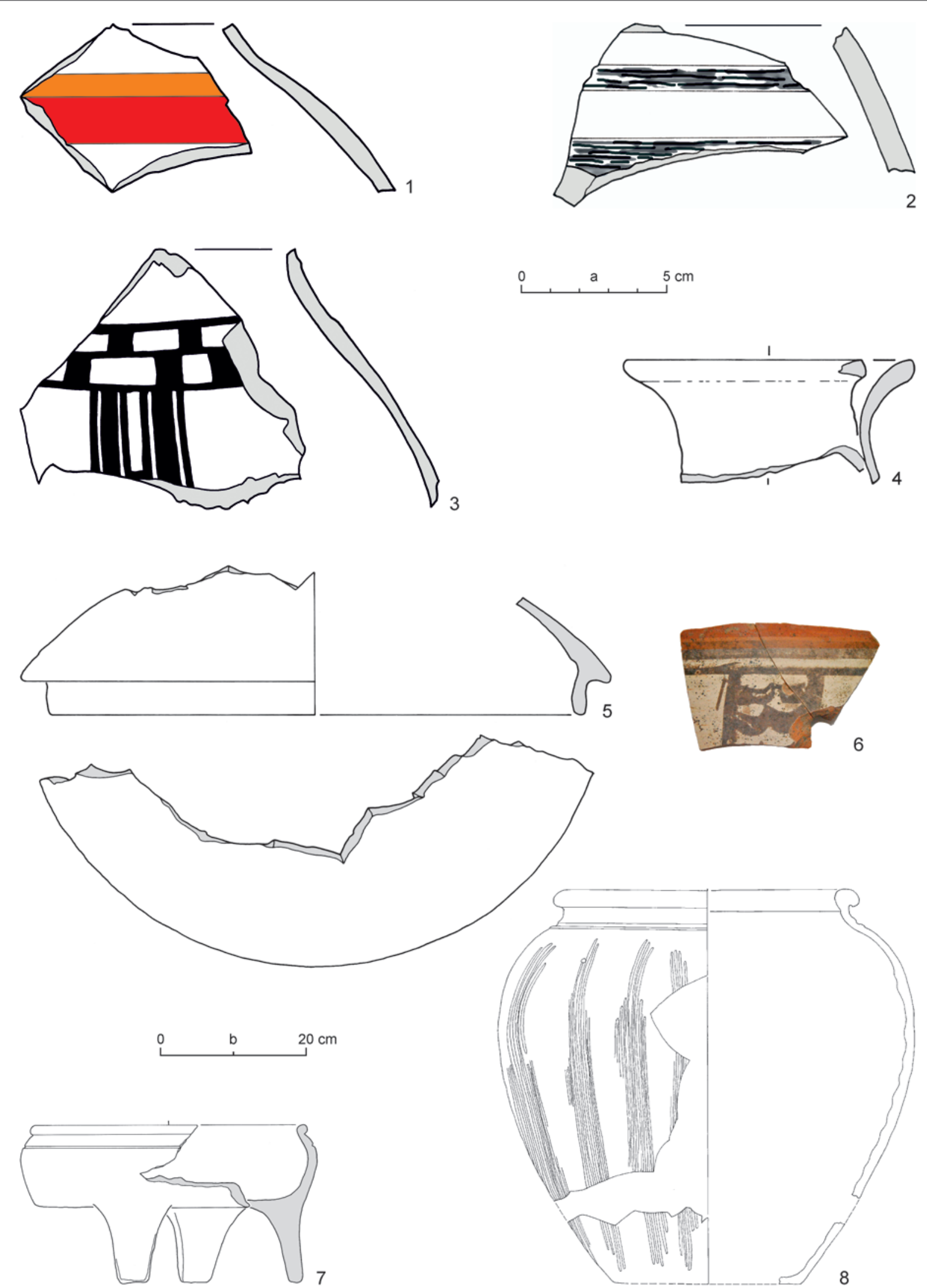

7

8

Taf. III. Wien III, Palais Rasumofsky, latènezeitliches Grubenhaus. Keramikfunde. Maßstab: a - 1-6; b - 7, 8. 


\section{LITERATUR}

Adler-Wölfl 2012 - K. Adler-Wölfl: Spätlatènezeitliche Fundstellen in Wien. Zborník SNM 106. Archeológia 22, 2012, 166-185.

Adler-Wölfl/Mosser 2015 - K. Adler-Wölfl/M. Mosser: Archäologie am Rochusmarkt. Die Grabungen in Wien 3, Rasumofskygasse 29-31. Fundort Wien 18, 2015, 4-48.

Artner 1998-1999 - W. Artner: Der Frauenberg bei Leibnitz, Steiermark, in der Spälatènezeit und in der vorclaudischen Kaiserzeit. Archaeologia Austriaca 82-83, 1998-1999, 221-341.

Bednár/Březinová/Ptáčková 2005 - P. Bednár/G. Březinová/ S. Ptáčková: Neskorolaténske osídlenie hradného návršia v Nitre. Študijné zvesti Aú SAV 37, 2005, 115-186.

Bónis 1969 - E. S. Bónis: Die spätkeltische Siedlung GellérthegyTabán in Budapest. Archaeologia Hungarica, S. N. 47. Budapest 1969.

Březinová 2000 - G. Březinová: Nitra-Šindolka. Siedlung aus der Latènezeit. Katalog. Archeologica Slovaca Monographiae. Catalogi 7. Nitra 2000.

Březinová/Chropovský 2020 - G. Březinová/B. Chropovský: Sídliská $z$ doby laténskej v Nitre. Archaeologica Slovaca Monographiae. Fontes 27. Nitra 2020.

Čambal 2004 -R. Čambal: Bratislavský hradný vrch-akropola neskorolaténskeho oppida. Zborník SNM. Archeológia. Suplementum 1. Bratislava 2004.

Čambal et al. 2006 -R. Čambal/M. Gregor/T. Krampl/P. Nagy: Neskorolaténske objekty v Bratislave na Cajkovského ulici č. 9. Zborník SNM 100. Archeológia 16, 2006, $123-160$.

Čambal et al. 2015 - R. Čambal/I. Bazovský/B. Kovár/ M. Buda: Boische Besiedlung im Oppidum von Bratislava und seinem Hinterland. In: M. Karwowski/V. Salač/S. Sievers (Hrsg.): Boier zwischen Realität und Fiction. Akten des internationalen Kolloquiums in Český Krumlov vom 14.-16. 11. 2013. Römisch-germanische Kommision des Deutschen Archäologischen Instituts. Kolloquien zur Vor- und Frühgeschichte 21. Bonn 2015, 225-242.

Čambal et al. 2016 - R. Čambal/M. Gregor/I. Bazovský/ G. Březinová/B. Kovár/M. Karwowski: The Pottery with Thickened Club Rim at the End of the Late La Tène Period in the Middle Danube Region (Mineralogical and Petrographic Characteristics). In: M. Karwowski/ P. C. Ramsl (eds.): Boii - Taurisci. Proceeding of the Internatiional Seminar. Oberleis-Klement, June 14 $4^{\text {th }}-15^{\text {th }} 2012$. Mitteilungen der Praehistorischen Kommission 85. Wien 2016, 147-169.

Čambal/Gregor 2008 - R. Čambal/M. Gregor: Engoby a pigmenty neskorolaténskej keramiky z bratislavského oppida. Zborník SNM 102. Archeológia 18, 2008, 95-110.

Čambal/Kovár 2019 - R. Čambal/B. Kovár: Značky na nádobách z doby laténskej na príklade lokality Bratislava-Vydrica. In: I. Bazovský/G. Březinová (eds.): L’udia a hory - archeologická perspektíva. Interakcie l'udských spoločenstiev horských a podhorských oblastí západného Slovenska. Zborník SNM. Archeológia. Suplementum 12. Bratislava - Nitra 2019, 179-188.

Čambal/Kovár/Hanuš 2012 - R. Čambal/B. Kovár/M. Hanuš: Typológia neskorolaténskej keramiky z Bratislavy-Vydrice. In: G. Březinová/V. Varsik (eds.): Archeológia na prahu histórie. Kživotnému jubileu Karola Pietu. Archaeologica Slovaca Monographiae. Communicationes 14. Nitra 2012, 117-136.
Čambal/Mináč/Zachar 2010 - R. Čambal/V. Mináč/L. Zachar: Laténske objekty 3/78 a 7/78 zo Senca-Svätého Martina. In: J. Beljak/G. Březinová/V. Varsik (eds.): Archeológia barbarov 2009. Hospodárstvo Germánov, sídliskové a ekonomické štruktúry od neskorej doby laténskej po včasný stredovek. Zborník referátov z 5. protohistorickej konferencie. Nitra 21.-25. septembra 2009. Archaeologica Slovaca Monographiae. Communicationes 10. Nitra 2010, 131-154. Čižmář 2003 - M. Čižmář: Laténské sídliště v Bořitově. Pravěk. Supplementum 10. Brno 2003.

Čižmář/Meduna 1985 - M. ČižmářrJ. Meduna: Značky na dnech laténské keramiky na Moravě. Památky archeologické 76, 1985, 78-100.

Cumberpatch 1993 - C. G. Cumberpatch: The Circulation of Late La Tène Slip Decorated Pottery in Slovakia, Southern Poland and Transdanubian Hungary. Slovenská archeológia 41, 1993, 59-81.

Donat/Pichler/Sedlmayer 2002 - P. Donat/E. Pichler/ H. Sedlmayer Aspekte spätkeltische und frührömischer Siedlungsentwicklung in Wien-Landstraße. Fundort Wien 5, 2002, 76-100.

Girardis 1937 - M. Girardi: Das Palais Rasumofsky. Geschichte und Schicksale eines Alt-Wiener Palastes. Wien 1937.

Hlava 2008 - M. Hlava: Grafit v době laténské na Moravě. Památky archeologické 99, 2008, 189-258.

Hlava/Vích 2007 - M. Hlava/D. Vích: Laténské osídlení Boskovicka. In: M. Hlava/D. Vích: Laténské osídleni Boskovicka. Laténské osídlení Uničovska. Českomoravské pomezí v době ř́mské. Pravěk. Supplementum 17. Brno 2007, 11-86.

Janšák 1955 - Š. Janšák: Hrnčiarska dielňa z neskorého laténu v Bratislave. Slovenská archeológia 3, 1955, 195-221.

Käferle/Schön 2011a - D. Käferle/D. Schön: Grabungsbericht Archäologie Service, Palais Rasumofsky. Wien 2011. Maßnahmennummer 01006.11.4. Unveröffentlicht.

Käferle/Schön 2011b - D. Käferle/D. Schön: KG Landstraße, 3. Bezirk. Fundberichte aus Österreich 50, 2011, 457, 458.

Kappel 1969 - I. Kappel: Die Graphittonkeramik von Manching. Ausgrabungen in Manching 2. Wiesbaden 1969.

Karl 1996 - R. Karl: Latènezeitliche Siedlungen in Niederösterreich. Untersuchungen zu Fundtypen, Keramikchronologie, Bautypen, Siedlungstypen und Siedlungsstrukturen im latènezeitlichen Niederösterreich. Historica Austria. Band 2-3. Wien 1996.

Maier 1970 - F. Maier: Die Bemalte Spätlatène-Keramik von Manching. Ausgrabungen in Manching 3. Wiesbaden 1970.

Meduna 1980 - J. Meduna: Die latènezeitlichen Siedlungen in Mähren. Praha 1980.

Musilová/Lesák 1996 - M. Musilová/B. Lesák: Neskorolaténske osídlenie na Hlavnom námestí č. 7. v Bratislave. Zborník SNM 90. Archeológia 6, 1996, 87-105.

Pieta 1996 - K. Pieta: Römische Import der Spätlatènezeit in der Slowakei. Arheloški vestnik 47, 1996, 183-195.

Pieta/Zachar 1993 - K. Pieta/L. Zachar: Neskorolaténske oppidum v historickom jadre mesta. In: T. Štefanovičová (zost.): Najstaršie dejiny Bratislavy. Bratislava 1993, 148-209.

Saliari/Pucher/Ramsl 2015 - K. Saliari/E. Pucher/P. C. Ramsl: Römische Haustiere eines Latènezeitlichen Hausherrn in Vindobona (Wien)? Beiträge zur Archäozoologie und Prähistorischen Anthropologie 10, 2015, 71-78. 
Timár 2016 - L. Timár: The Sunken-Featured Buildings of the Middle La Tène Period: Problems and Reconstructions. In: M. Karwowski/P. C. Ramsl (eds.): Boii - Taurisci. Proceedings of the International Seminar. Oberleis-Klement, June $14^{\text {th }}-15^{\text {th }}$, 2012. Mitteilungen der Prähistorischen Kommission 85. Wien 2016, 191-203.

Trebsche 2010 - P. Trebsche: Auswertung der latènezeitlichen Befunde und Funde von Michelstetten. In: E. Lauermenn (Hrsg.): Die latènezeitliche Siedlung von Michelstetten. Die Ausgrabungen des Niederösterreichischen Museums für Urgeschichte in den Jahren 1994-1999. Archäologische Forschungen in Niederösterreich 7. St. Pölten 2010, 15-115.

Trebsche 2011 - P. Trebsche: Eisenzeitliche Graphittonkeramik im mittleren Donauraum. In: K. Schmotz (Hrsg.): Vorträge des 29. Niederbayerischen Archäologentages. Rahden/Westf. 2011, 449-481.

Urban 1996 - O. H. Urban: Zur Chronologie der jüngeren Latènezeit in Ostösterreich. Arheloški vestnik 47, 1996, 197-207.

Manuskript angenommen am 16. 6. 2021

Abstract translated by Peter C. Ramsl

Priv. Doz. Mag. Dr. Peter C. Ramsl

Institut für Urgeschichte und Historische Archäologie

Franz-Klein-Gasse 1

A - 1190 Wien

peter.ramsl@univie.ac.at
Urban 1999 - O. H. Urban: Der Leopoldsberg. Archäologische Forschungen auf dem Wiener Hausberg. Wiener archäologische Studien. Band 2. Wien 1999.

Vrtel 2012 - A. Vrtel: Keltské oppidum v Bratislave. In: J. Šedivý/T. Štefanovičová (zost.): Dejiny Bratislavy 1. Od počiatkov do prelomu 12. a 13. storočia. Brezalauspurc na križovatke kultúr. Bratislava 2012, 164-179.

Waldhauser u. AG 1993 - J. Waldhauser und Arbeitsgruppe: Die hallstatt- und laténzeitliche Siedlung mit Gräberfeld bei Radovesice in Böhmen. Archeologický výzkum v severních Čechách 21. Praha 1993.

Zachar 1981 - L. Zachar: Neskorolaténske vrstvy na Partizánskej ulici v Bratislave. Zborník SNM 75. História 21, 1981, 35-54.

Zachar 1982 - L. Zachar: Príspevok k problematike bratislavského oppida. Zborník SNM 76. História 22, 1982, 31-49.

Zachar/Rexa 1988 - L. Zachar/D. Rexa: Beitrag zur Problematik der spätlatènezeitlichen Siedlungshorizonte innerhalb des Bratislavaer Oppidums. Zborník SNM 82. História 28, 1988, 27-72.

Mgr. Radoslav Čambal, PhD.

SNM - Archeologické múzeum

Žižkova 12

P. O. BOX 13

SK - 81006 Bratislava

radoslav.cambal@snm.sk 